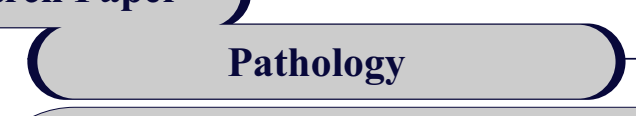

\title{
DURAL INTRACRANIAL PLASMA CELL NEOPLASMS MIMICKING MENINGIOMA
}

\begin{tabular}{ll}
\hline Dr. Surabhi Tyagi* & $\begin{array}{l}\text { M.D.Pathology, Prof. Neuropathology, Department of Pathology, Mahatma Gandhi } \\
\text { Medical College, Jaipur, Rajasthan.*Corresponding Author }\end{array}$ \\
\hline Dr. Sharma B. S & $\begin{array}{l}\text { Mch Neurosurgery, Prof and HOD Neurosurgery, Director Neurosciences, Mahatma } \\
\text { Gandhi Medical College, Jaipur, Rajasthan. }\end{array}$ \\
\hline $\begin{array}{l}\text { Dr. Kalla Amrit } \\
\text { Raj }\end{array}$ & $\begin{array}{l}\text { III rd year M.D. Resident ,Department of Pathology, Mahatma Gandhi Medical } \\
\text { College, Jaipur, Rajasthan. }\end{array}$ \\
\hline Dr. Chowdhary Jai & $\begin{array}{l}\text { Assistant Professor, Department of Radiology, Mahatma Gandhi Medical College, } \\
\text { Jaipur, Rajasthan }\end{array}$
\end{tabular}

ABSTRACT Meningioma is the most common Dural based tumours of CNS. Plasma cell neoplasms rarely involve the CNS as Dural lesions and occasionally can arise from calvarium and skull base. They are characterized by clonal proliferation of plasma cells and have a benign course, being radiosensitive, unless associated with multiple myeloma. On imaging, the differential diagnosis is meningioma, metastasis, lymphoma, osteochondroma, infectious meningitis, plasma cell granuloma and sarcoma of the dura .We present a 70 year old male with extramedullary lesion in right parasagittal region mimicking meningioma on MRI and intraoperatively. Histopathological diagnosis was a solitary plasmacytoma. There was no evidence of multiple myeloma on complete work up. Intracranial plasmacytoma must be suspected in all patients with a solitary Dural mass, and in the differential diagnosis of meningeal tumours. The parasagittal location of the tumour and its extramedullary nature indicates a favourable prognosis and rare chances of progression to multiple myeloma.

KEYWORDS : Dural based lesions, plasma cell neoplasms, meningiomas, extramedullary plasmacytoma

\section{INTRODUCTION}

Plasma cell neoplasms arise from proliferation of a single clone of B cell lymphocytes, $5 \%$ of which present as solitary lesions of bone and soft tissue. We present a case of intracranial extramedullary Dural based plasma cell neoplasm with radiological and intraoperative diagnosis as meningioma. Patient presented to our neurosurgical services with a midline parietal intradiploic SOL eroding the parietal bone with dura and scalp and invading the sinus

\section{Case report-}

A 70 year old male presented to our neurosurgical services, with a history of recurrent headache, gradually progressive scalp swelling, numbness and vertigo for 1 year. CT scan revealed an expansile osteolytic lesion measuring $6 \times 4.4 \times 4.7 \mathrm{~cm}$ in the right high parasagittal region with intracranial extra-axial extension causing scalloping of underlying brain parenchyma \& subcutaneous scalp with multiple bony spicules. Fig [1A, B, C].
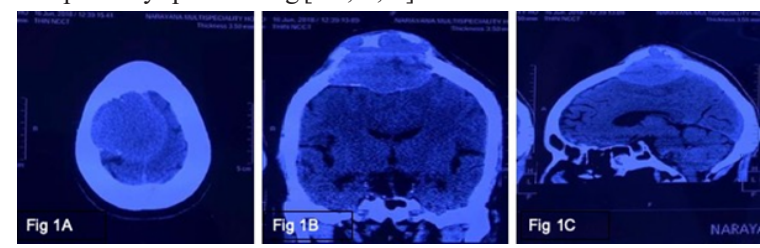

Fig 1 A,B,C - NCCT scan showing extra-axial mass involving parietal bones with intra - \& extra - cranial extension T2W MRI showed the lesion to be iso- to hyper- intense, involving the adjacent skull bones, scalp \& superior sagittal sinus. The differential diagnosis considered were atypical meningioma, metastasis or a primary skull bone tumor. [Fig 2A, B, C]


Fig 2 A,B,C - T2 weighted MRI images showing predominantly solid mass lesion with central necrotic areas involving parietal bone with complete destruction of calvarium extending into scalp and extradural region of high parietal area. Brain parenchyma is compressed, however, no invasion is seen.
Intraoperatively tumor was attached to dura, eroded right parietal bone and scalp.

Routine H\&E examination of the biopsy revealed sheets of atypical plasma cells invading the bony trabeculae. The cells were round to oval having abundant cytoplasm and eccentric nuclei with some binucleate forms. The nuclear cytoplasmic ratio was increased with prominence of nucleoli. The mitotic activity was brisk. On immunohistochemistry (IHC), the tumor was positive for CD138, EMA and vimentin. Kappa expression is restricted. [Fig.3A, B, C, D, E]



Fig 3. Shows a cellular plasma cell neoplasm composed of sheets of atypical plasma cells including binucleate cells and increased mitosis ( arrow ) [A], Tumor cells are diffusely positive for CD 138 [B], Kappa expression is restricted $[\mathrm{C}]$, and it is predominantly negative for lambda except for patchy positivity[D].The tumor exibits high MIB1 labelling index [E]. All magnifications are indicated in the scale bar of individual figures.

The diagnosis of Dural plasmacytoma with bone involvement was made. A bone marrow (BM) aspiration revealed $4.6 \%$ plasma cells (slightly higher from reference range of $0-1.4 \%$ ) and the BM biopsy showed a hyper cellular marrow with mild interstitial plasmacytosis.

Serum protein electrophoresis did not reveal monoclonal antibody and urine test for Bence Jones Protein was negative. There was no abnormal FDG uptake on F18-FDG PET scan.

Taking together these findings, our case conformed to the criteria of solitary bone plasmacytoma with minimal bone involvement. ( $4.6 \%$ ) 


\section{DISCUSSION -}

Solitary Dural plasmacytoma is rare and mainly occurs in older individuals with female preponderance of about $89 \%{ }^{\left[{ }^{[1]}\right.}$ Our case was an elderly male aged 70 years. As there is no pathognomonic radiological feature for its diagnosis, the differential diagnosis of a Dural plasmacytoma includes meningioma, metastasis, lymphoma ,osteochondroma, infectious meningitis ,plasma cell granuloma and sarcoma of the dura. ${ }^{[2][3][4]}$ In our case the radiological differential diagnosis considered were atypical meningioma, metastasis or a primary skull bone tumor.

Intraoperatively the tumor was attached to dura, eroded right parietal bone and scalp and the diagnosis was meningioma ${ }^{[3]}$. Cranial and intracranial plasmacytomas may involve the cranial vault, skull base or brain parenchyma though intracranial involvement is less common. ${ }^{[1]}$

In our case there was extension to adjacent skull bones, scalp and the superior sagittal sinus, scalloping of the underlying brain parenchyma. Plasma cell neoplasms are characterized by monoclonal proliferation of plasma cells. ${ }^{[4]}$

Solitary plasmacytoma is a single lesion without any evidence of myeloma cells in marrow or in any other part of the body. Primary extramedullary plasmacytomas are not common and account for only $4 \%$ of all plasma cell tumors. They may be extramedullary or intramedullary and may progress to multiple myeloma or may be associated with it. ${ }^{[4]}$ It has been shown that conversion to multiple myeloma occurs mainly in intramedullary plasmacytomas.

Higurashi et al reported the indicators predicting the progression of plasmacytoma to multiple Myeloma which included the intramedullary location, presence of residual tumor and skull base location. ${ }^{[5]}$

The authors reported that $60-70 \%$ of intramedullary lesions arising from bone progress to MM, in comparison to only $10 \%$ to $20 \%$ of extramedullary lesions arising from soft tissues of paranasal sinuses, mastoid and middle ear epithelium. Many skull base lesions which were initially considered solitary eventually progressed to MM ,so it was suggested that as most plasma cell myelomas of the skull base are intramedullary and are in the early stage of MM. On the contrary, plasma cell myelomas of the other cranial locations are mostly extramedullary and likely to be solitary plasmacytomas.

A similar study by Chiang et al on 11 plasmacytoma cases reported that solitary plasmacytoma arises from medullary tissue as an early manifestation of multiple myeloma and within 3 to 5 years progresses to multiple myeloma with about $50 \%$ of the cases disseminating to the skeletal system. ${ }^{[6]}$

Schwartz et al also reported similar results of intramedullary plasmacytoma progressing to multiple myeloma more frequently $(50 \%)$ in comparison to extramedullary plasmacytomas $(30 \%)$ and stated that cranial base location was the strongest predictor for development of multiple myeloma. ${ }^{[1]}$

According to Cerase et al, the intracranial involvement in multiple myeloma is from extramedullary neoplasms arising from calvaria, skull base or nasal and paranasal sinuses Dural involvement was the due to the direct spread of contiguous bone lesions and leptomeningeal spread due to hematogenous route. ${ }^{[7]}$

On the contrary,Du Preez JH et al demonstrated that, of 18 calvarial and 13 dural-based lesions, only 2 calvarial lesions progressed to MM. ${ }^{[8]}$ MM may take many years to develop from a plasmacytoma extracranially. ${ }^{[1]}$

Our case was an extramedullary plasmacytoma with no evidence of multiple myeloma after complete workup. The location of the tumor being in the parasagittal region and extramedullary in nature has favourable prognosis and rare chances of progression to multiple myeloma.

The ideal treatment plan is complete surgical resection, if anatomically suitable with postoperative radiotherapy ${ }^{[7]}$ which is very effective leading to complete disappearance of the mass ${ }^{[2]}$ because of the radiosensitive nature of the tumor Our patient also received adequate radiotherapy after surgery. There was no recurrence after postoperative radiotherapy.
To conclude intracranial plasmacytoma must be suspected in all patients with a solitary dural mass and in the differential diagnosis of meningeal tumours, definitive diagnosis requires the examination of histopathology specimen. Monoclonal nature of the tumor should also be determined to rule out inflammatory pathology like plasma cell granuloma.

\section{REFERENCES}

1. Schwartz TH, Rhiew R, Isaacson SR, Orazi A, Bruce JN. Association between intracranial plasmacytoma and multiple myeloma: clinicopathological outcome study. Neurosurgery 2001;49(5): 1039-44; discussion 1044-5.

2. Manabe M, Kanashima H, Yoshii Y, Mukai S, Sakamoto E, Iwai Y, Kubo Y, Fukushima $\mathrm{H}$, Inoue T, Teshima H: Extramedullary plasmacytoma of the dura mimicking meningioma. Int J Hematol 91:731-732,2010

3. Sahin F, Saydam G, Ertan Y, Calli C, Dönmez A, Tombuloglu M: Dural plasmacytoma mimicking meningioma in a patient with multiple myeloma. J Clin Neurosci 13:259261,2006

4. Negar azarpıra, puria NOShaD1, Sara pakBaz, Simin TOraBıNEghaD, mohamad rakE1, akbar Safai Dural Plasmacytoma mimicking meningioma. Turkish neurosurgery.

5. Higurashi M, Yagishita S, Fujitsu K, Kitsuta Y, Takemoto Y, Osano S. Plasma cell myeloma of the skull base: report of two cases. Brain Tumor Pathol 2004;21(3):135-41.

6. Chiang SK, Canalis RF, Ishiyama A, Eversole LR, Becker DP. Plasmacytoma of the temporal bone. Am J Otolaryngol 1998; 19(4):267-73.

7. Cerase A, Tarantino A, Gozzetti A, Muccio CF, Gennari P, Monti L et al. Intracranial involvement in plasmacytomas and multiple myeloma: a pictorial essay. Neuroradiology. 2008;50(8):665-74.

8. Du Preez JH, Branca EP. Plasmacytoma of the skull: case reports. Neurosurgery 1991; 29(6):902-6 